\title{
Orbital Engineering in Symmetry-Breaking Polar Heterostructures
}

\author{
Ankit S. Disa, ${ }^{1,2,{ }^{*}}$ Divine P. Kumah, ${ }^{1,2}$ Andrei Malashevich, ${ }^{1,2}$ Hanghui Chen, ${ }^{1,2,3,4}$ Dario A. Arena, ${ }^{5}$ Eliot D. Specht, ${ }^{6}$ \\ Sohrab Ismail-Beigi, ${ }^{1,2,7}$ F. J. Walker, ${ }^{1,2}$ and Charles H. Ahn ${ }^{1,2,7}$ \\ ${ }^{1}$ Center for Research on Interface Structures and Phenomena, Yale University, New Haven, Connecticut 06511, USA \\ ${ }^{2}$ Department of Applied Physics, Yale University, New Haven, Connecticut 06511, USA \\ ${ }^{3}$ Department of Physics, Columbia University, New York, New York 10027, USA \\ ${ }^{4}$ Department of Applied Physics and Applied Mathematics, Columbia University, New York, New York 10027, USA \\ ${ }^{5}$ National Synchrotron Light Source, Brookhaven National Laboratory, Upton, New York 11973, USA \\ ${ }^{6}$ Materials Science and Technology Division, Oak Ridge National Laboratory, Oak Ridge, Tennessee 37831, USA \\ ${ }^{7}$ Departments of Physics and Mechanical Engineering and Materials Science, Yale University, New Haven, Connecticut 06511, USA
}

(Received 14 November 2014; published 12 January 2015)

\begin{abstract}
We experimentally demonstrate a novel approach to substantially modify orbital occupations and symmetries in electronically correlated oxides. In contrast to methods using strain or confinement, this orbital tuning is achieved by exploiting charge transfer and inversion symmetry breaking using atomically layered heterostructures. We illustrate the technique in the $\mathrm{LaTiO}_{3}-\mathrm{LaNiO}_{3}-\mathrm{LaAlO}_{3}$ system; a combination of x-ray absorption spectroscopy and $a b$ initio theory reveals electron transfer and concomitant polar fields, resulting in a $\sim 50 \%$ change in the occupation of $\mathrm{Ni} d$ orbitals. This change is sufficiently large to remove the orbital degeneracy of bulk $\mathrm{LaNiO}_{3}$ and creates an electronic configuration approaching a single-band Fermi surface. Furthermore, we theoretically show that such three-component heterostructuring is robust and tunable by choice of insulator in the heterostructure, providing a general method for engineering orbital configurations and designing novel electronic systems.
\end{abstract}

Achieving new electronic orbital configurations in solids can lead to novel electronic and magnetic phenomena, especially in systems with numerous competing quantum phases, such as transition-metal oxides. Examples of properties that depend on orbital configuration include topological surface states [1], oxygen electrocatalysis [2], interface magnetism between nonmagnetic materials [3], metallic quantum well states [4], tunable pseudogap phases [5], high-temperature superconductivity [6], and control of the Mott metal-insulator transition [7].

For transition-metal oxides (TMOs), orbital manipulation plays an essential role in understanding and controlling the diverse range of observed behaviors because the electronically active $d$ orbitals of the transition-metal cations underpin a strong coupling between electronic and structural degrees of freedom [8,9]. For near-cubic symmetry, these orbitals are split into levels with $t_{2 g}$ and $e_{g}$ symmetry. We focus here on controlling the orbital properties in materials where the lower-energy $t_{2 g}$ levels are full, leaving only the two $e_{g}$ orbitals (the $3 z^{2}-r^{2}$ and $x^{2}-y^{2}$ orbitals) active near the Fermi energy. Much of the distinctive physics of TMOs, such as colossal magnetoresistance in the manganites [10], spin-state transitions in cobaltates [11], and high-temperature superconductivity in the cuprates [6,12], occurs in such systems. The electronic properties of these materials depend sensitively on their orbital structure, including the relative energies of the orbitals, their filling, and their intermixing [13]. In particular, confining the valence electrons to a single orbital or energy band enhances quantum correlations and phase competition by reducing available degrees of freedom.

We consider the rare-earth nickelates $\left(R \mathrm{NiO}_{3}\right)$ [14-20] as a model system to demonstrate orbital engineering. In the bulk, the nominal ionic configuration of the $\mathrm{Ni}^{3+}$ is $d^{7}$ containing a full $t_{2 g}$ shell and a single electron occupying the twofold degenerate $e_{g}$ manifold. We focus on $\mathrm{LaNiO}_{3}$, the most metallic of the $R \mathrm{NiO}_{3}$ family, which has a pseudocubic perovskite structure with an octahedral coordination that splits the $t_{2 g}$ band but does not affect the degeneracy of the $e_{g}$ band. Significant effort has been expended over the last several years to achieve a large orbital polarization in $\mathrm{LaNiO}_{3}$-based structures, with the goal of isolating and achieving half filling of the $x^{2}-y^{2}$ band, in analogy to the cuprates [21,22]. Previous proposals for breaking orbital degeneracy in this system by using quantum confinement and/or strain result in orbital polarizations that are small or modest [23-27]: to date, the largest experimentally observed effect has resulted in an $e_{g}$ splitting of $\sim 300 \mathrm{meV}$ and $<20 \%$ change in orbital occupancy compared to the bulk [28].

Here, we describe and experimentally demonstrate a novel method to artificially transform an orbitally degenerate $e_{g}$ system into a single band system by using atomiclayer synthesis to drastically alter its symmetry and filling. Our experimental work is based on a new approach that utilizes atomically layered heterostructures to yield a large orbital polarization and a nondegenerate band structure 
$[29,30]$. This proposal takes advantage of inversion symmetry breaking in three-component heterostructures, which has previously been shown to lead to enhanced ferroelectric polarization [31-33]. The large orbital modification we achieve takes advantage of such heterostructuring and uses robust general physical mechanisms, in particular, the combination of inversion symmetry breaking, charge transfer, and polar structural distortions. The relative complexity of these structures, however, is a challenge for experimental realization $[34,35]$. We describe here the fabrication and properties of such three-component heterostructures, and we experimentally demonstrate that they exhibit large orbital polarizations ( $50 \%$ change in orbital occupancy compared to the bulk) as a result of charge transfer and polar fields. The realization of this widely tunable structure opens up the opportunity to apply these materials design principles to other systems in which one wishes to study or engineer orbital configuration to influence orbitally induced behavior.

We fabricate nickelate superlattices on $\mathrm{LaAlO}_{3}(001)$ single crystal substrates using oxygen plasma-assisted molecular beam epitaxy (MBE). The layering sequences for the two- and three-component superlattices are $\left[\left(\mathrm{LaNiO}_{3}\right)_{3}-\left(\mathrm{LaAlO}_{3}\right)_{6}\right] \times 20$ and $\left[\left(\mathrm{LaTiO}_{3}\right)_{1}-\left(\mathrm{LaNiO}_{3}\right)_{1}-\right.$ $\left.\left(\mathrm{LaAlO}_{3}\right)_{3}\right] \times 12$, and will be henceforth referred to as LNO-LAO and LTNAO, respectively. X-ray absorption (XAS) and x-ray linear dichroism (XLD) measurements are carried out at the National Synchrotron Light Source (NSLS) at beamline U4B. Atomic-scale structure determination is performed by measuring crystal truncation rods (CTR) using high-resolution synchrotron $\mathrm{x}$-ray diffraction at the NSLS using beamline X21 and the Advanced Photon Source using beamline 33ID. Density functional theory (DFT) calculations for nickelate structures are performed within the local density approximation using the plane wave pseudopotential approach, as implemented in QUANTUM ESPRESSO with ultrasoft pseudopotentials [36]. Additional methodological details can be found in Ref. [37].

The DFT calculations show that in contrast to bulk $\mathrm{LaNiO}_{3}$ or LNO-LAO superlattices, which display degenerate band structures with equally or nearly equally wide $\mathrm{Ni}$ $d$ bands of $x^{2}-y^{2}$ and $3 z^{2}-r^{2}$ character crossing the Fermi level [Figs. 1(a) and 1(b)], the lower symmetry in the LTNAO superlattice yields a nondegenerate band structure [Fig. 1(c)]. This is exemplified by the fact that the Fermi level cuts through the center of a wide band of primarily $x^{2}-y^{2}$ character, while a narrow band of primarily $3 z^{2}-r^{2}$ symmetry lies almost entirely below the Fermi level. This asymmetry of the $\mathrm{Ni} e_{g}$ states is due to a structural asymmetry around the $\mathrm{Ni}$ in the three-component superlattice, whereby the apical Ni-O bonds are elongated due to internal polar fields pointing towards the Ni [29]. These polar fields emerge as a result of unidirectional electron transfer from $\mathrm{Ti}$ to $\mathrm{Ni}$, which also serves to dope the $3 z^{2}-r^{2}$ band. The combination of inversion symmetry breaking, charge transfer, and internal polar fields forms the basis for the orbital modification in the LTNAO superlattice, and we verify these key properties here.

First, we demonstrate charge transfer from $\mathrm{Ti}$ to $\mathrm{Ni}$ that is predicted for LTNAO superlattices [50] and shown in Fig. 2(a) as calculated using DFT [37]. The primary effect is the transfer of an electron from the $\mathrm{Ti} t_{2 g}$ states to the $\mathrm{Ni}$ $3 z^{2}-r^{2} e_{g}$ state through the intermediary apical oxygen. In bulk, the Ti and $\mathrm{Ni}$ ions in $\mathrm{LaTiO}_{3}$ and $\mathrm{LaNiO}_{3}$ are in the $3+$ oxidation state. After the electron transfer in the heterostructure, the oxidation states should change such that $\mathrm{Ti}^{3+} \rightarrow \mathrm{Ti}^{4+}$ and $\mathrm{Ni}^{3+} \rightarrow \mathrm{Ni}^{2+}$. In order to confirm this transfer, we perform XAS measurements at the Ti $L$ edge on the MBE-grown LTNAO superlattice. Comparing the XAS spectrum of the LTNAO to those of bulk specimens, in which the $\mathrm{Ti}$ atoms have the $4+\left(\mathrm{SrTiO}_{3}\right)$ and $3+\left(\mathrm{LaTiO}_{3}\right)$ oxidation states, we observe in Fig. 2(b) that the superlattice contains predominantly $\mathrm{Ti}^{4+}$. XAS measurements of the Ni $K$ edge also show a shift relative to bulk $\mathrm{LaNiO}_{3}$ and LNO-LAO superlattices, corresponding to $\mathrm{Ni}$ ions being in the expected $2+$ state in the LTNAO structure [37]. Thus, both the $\mathrm{Ti}$ and $\mathrm{Ni}$ formal valence states in the LTNAO display the charge transfer of a full electron from $\mathrm{Ti}$ to $\mathrm{Ni}$.

The effect of this charge transfer on the resulting electronic structure of the LTNAO is twofold [29]. First, the transferred electron primarily occupies the $3 z^{2}-r^{2}$ band, providing a selective internal doping effect that
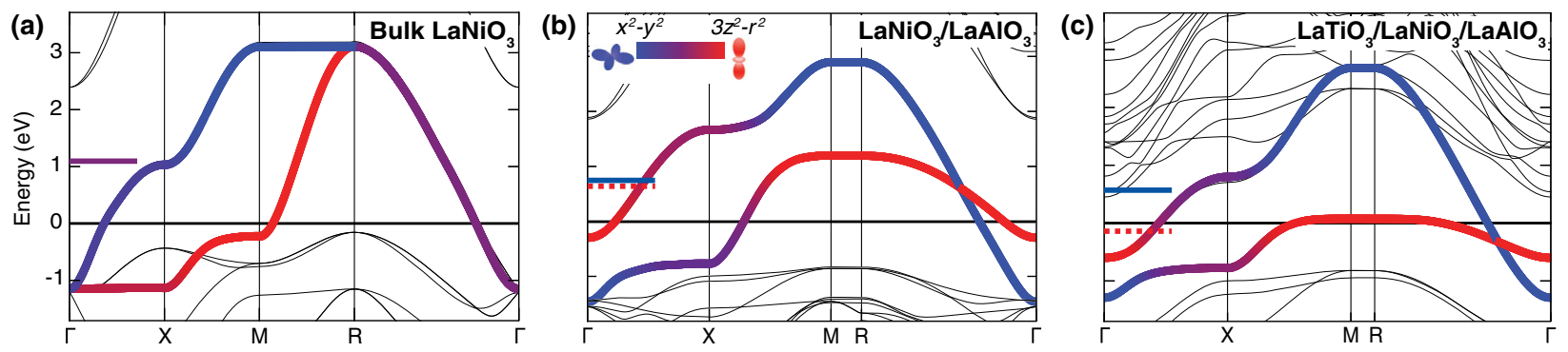

FIG. 1 (color online). Calculated $1 \times 1$ band structures for (a) bulk $\mathrm{LaNiO}_{3}$, (b) two-component, and (c) three-component superlattices near the Fermi level with projections onto Wannier states represented by bold color shading. Horizontal lines indicate centers of $3 z^{2}-r^{2}$ (red dashed) and $x^{2}-y^{2}$ (blue solid) bands. 
(a)

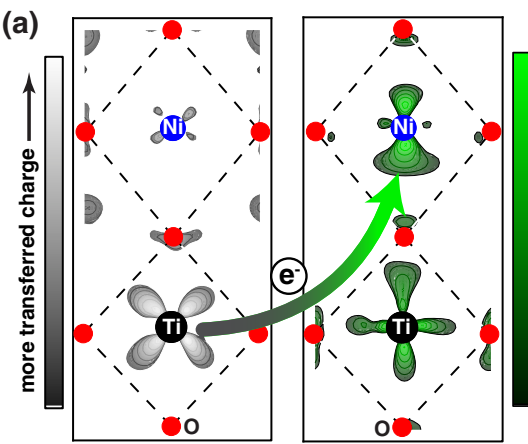

(b)
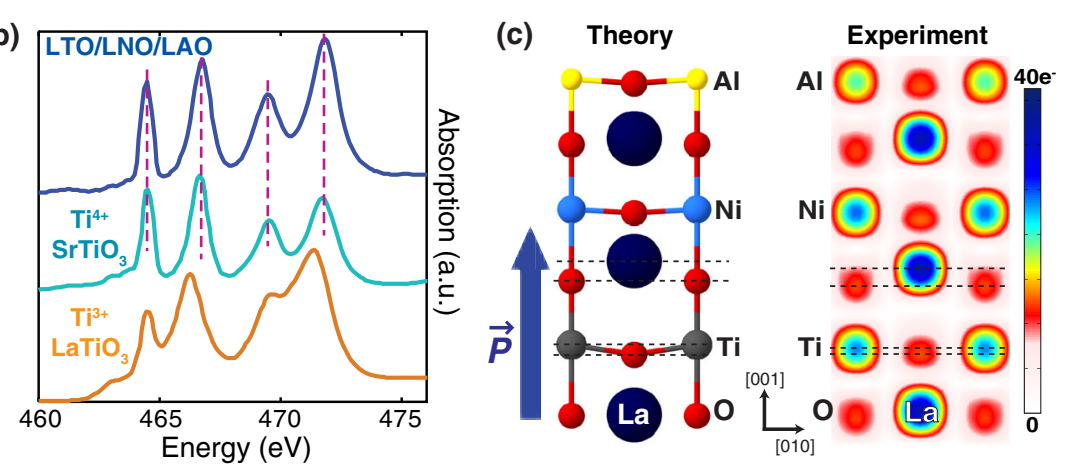

FIG. 2 (color online). (a) DFT-calculated electron transfer from Ti $t_{2 g}$ to Ni $e_{g}$ orbitals showing donor states (left) and acceptor states (right). Backbonding leads to some electron transfer from the Ni to lower-lying Ti levels. (b) XAS spectra for an MBE-grown LTNAO superlattice, bulk $\mathrm{SrTiO}_{3}$, and a thick $(10 \mathrm{~nm}) \mathrm{LaTiO}_{3}$ film grown on $\mathrm{LaAlO}_{3}$. Dashed lines show the approximate peak positions of the LTNAO spectrum, in agreement with a predominantly $\mathrm{Ti}^{4+}$ oxidation state. (c) Calculated atomic structure (left) and COBRA-derived electron density map (right) for a single-repeat LTNAO. Both calculated and measured structures show the atomic displacements due to the polar electric field $(\vec{P})$ that is driven by the electron transfer.

enhances the difference in orbital occupations. Second, the charge imbalance set up as a result of the electron transfer forms an internal dipole field driving polar distortions and apical Ni-O bond elongations in the LTNAO, which ultimately lead to splitting of the $e_{g}$ orbital energies. In this context, the experimental verification of the unidirectional transfer of a full electron should be highlighted: the observed magnitude of this interfacial charge transfer, which exceeds previous reports in oxide superlattices [13], and the asymmetry of the effect are crucial for producing large polar fields pointing towards each $\mathrm{NiO}_{2}$ layer, and hence, a large Ni orbital polarization.

Figure 2(c) shows the atomic structure of one repeat unit of LTNAO, as calculated from DFT. Polar displacements of the ions away from their ideal, planar geometry are evident (positively charged La and Ti ions move up and negatively charged $\mathrm{O}$ ions move down), leading to apical Ni-O bond elongation and a net dipole moment $\vec{P}$ directed towards the $\mathrm{Ni}$ from below. We experimentally determine the dipole moment from the ionic positions of $\mathrm{Ni}, \mathrm{O}$, and $\mathrm{Ti}$ [Fig. 2(c)], as determined using coherent bragg rod analysis (COBRA) of the CTRs [51] and their displacement away from an undistorted reference structure [37]. We find that the dipole moment points towards the $\mathrm{Ni}$ [Fig. 2(c)] and that the magnitude of the dipole moments determined from experiment and theory agree $\left(P_{\text {theory }}=2.93 \mathrm{e} \AA, P_{\text {expt }}=3.01 \mathrm{e} \AA\right)$. Structural analysis performed using periodic LTNAO superlattice structures shows similar agreement [37].

The ratio of apical to in-plane $\mathrm{Ni}-\mathrm{O}$ bond lengths $\left(d_{\text {ap }} / d_{\text {inp }}\right)$ serves as a metric for considering the modification of the crystal field caused by the polar distortions. There are two distinct apical Ni-O bonds per $\mathrm{LaNiO}_{3}$ layer in the LTNAO: one towards Ti and one towards Al. For the periodic superlattice, we find $d_{\text {ap }} / d_{\text {inp }}=1.14$ from theory and $d_{\text {ap }} / d_{\text {inp }}=1.06$ from experiment for the average of the two apical Ni-O bonds; the longer apical Ni-O bond gives $d_{\text {ap }} / d_{\text {inp }}=1.18$ and 1.16 , respectively (the experimental results average over all 12 repeats). We note that the long $d_{\text {ap }} / d_{\text {inp }}$ ratio corresponds to a large (volume-conserving) biaxial strain on the $\mathrm{LaNiO}_{3}$ layer of $\sim 8-10 \%$, which cannot feasibly be achieved using conventional epitaxial techniques that use lattice misfit to induce strain. As will be discussed below, this substantial modification of the crystal field environment, coupled with the internal electron doping, leads to a significant orbital polarization not realizable via epitaxial strain.

We next establish the achievement of a large orbital polarization in the LTNAO heterostructure. XLD delivers both element- and orbital-specific information by comparing polarization-dependent XAS spectra. In particular, at the Ni $L$ edge, transitions to the $x^{2}-y^{2}$ and $3 z^{2}-r^{2}$ orbitals can be individually excited by choosing the incident polarization to be in plane $\left(E_{x y}\right)$ or out of plane $\left(E_{z}\right)$ with respect to the sample surface. The integrated XLD spectra, $I_{E_{z}}$ and $I_{E_{x y}}$, provide a direct measurement of the ratio of $e_{g}$ holes [23,52]:

$$
r=\frac{h_{3 z^{2}-r^{2}}}{h_{x^{2}-y^{2}}}=\frac{3 I_{E_{z}}}{4 I_{E_{x y}}-I_{E_{z}}},
$$

where $r$ is the hole ratio and $h_{j}$ is the number of holes in the orbital $j$. For the case of creating a nondegenerate band structure with a filled $3 z^{2}-r^{2}$ band, the relevant limits for $r$ range from 0 to 1 . A value of $r$ close to 0 signifies a small degree of unoccupied states with $3 z^{2}-r^{2}$ character, while $r=1$ indicates fully degenerate $e_{g}$ bands. This quantity can also be determined from first principles band structure calculations by integrating the projected density of states (PDOS) for all $x^{2}-y^{2}$ and $3 z^{2}-r^{2}$ states above the Fermi level.

Another quantity explored in the literature is the "orbital polarization" $P$ defined as 


$$
P=\frac{n_{3 z^{2}-r^{2}}-n_{x^{2}-y^{2}}}{n_{3 z^{2}-r^{2}}+n_{x^{2}-y^{2}}}=\left(\frac{4}{n_{e_{g}}}-1\right) \frac{1-r}{1+r},
$$

where $n_{j}$ is the number of electrons occupying orbital $j$. We point out, as detailed in Ref. [35], that $r$ represents a practical observable for determining the orbital configuration that can be reliably compared to and predicted by $a b$ initio band structure methods. Unlike $P$, it does not sensitively depend on choice of local atomic basis or, equivalently, on $n_{e_{g}}$, the total occupancy of the $e_{g}$ manifold, which is an ill-defined quantity experimentally and theoretically $[28,35]$.

We use DFT to compute $r$ for bulk $\mathrm{LaNiO}_{3}, \mathrm{LNO}-\mathrm{LAO}$ superlattices, and LTNAO superlattices. As expected, bulk $\mathrm{LaNiO}_{3}$ [Fig. 1(a)] is fully degenerate with $r=1$. The effect of the two-component superlattice is to confine the $\mathrm{Ni}$ electrons and reduce the out-of-plane hopping. While this confinement narrows the $3 z^{2}-r^{2}$ band, the center energy of the $3 z^{2}-r^{2}$ band is shifted by only $\Delta_{e_{g}} \sim 0.1 \mathrm{eV}$ [Fig. 1(b)]. Thus, the integrated $3 z^{2}-r^{2}$ PDOS is nearly the same as for the bulk, with $r=0.92$. In contrast, due to the aforementioned structural distortions and charge transfer, the LTNAO superlattice [Fig. 1(c)] exhibits a narrow band of primarily $3 z^{2}-r^{2}$ character that is nearly full and split by $\Delta_{e_{g}} \sim$ $0.8 \mathrm{eV}$ from a partially filled band of primarily $x^{2}-y^{2}$ symmetry, yielding $r=0.48$. Hence, we find that the band degeneracy is largely broken, and the LTNAO superlattice is a nearly fully nondegenerate material that can be made, upon slight electron doping, to have a single band at the Fermi level. Electron doping may be achieved experimentally by substitution of $\mathrm{La}^{3+}$ for $\mathrm{Ce}^{3+/ 4+}$, either directly into the $\mathrm{LaNiO}_{3}$ layer or through remote doping from the $\mathrm{LaTiO}_{3}$ or $\mathrm{LaAlO}_{3}$ layers. The experimental feasibility of such approaches is the subject of ongoing study.

To measure the orbital polarization experimentally, we conduct XLD measurements on the MBE-grown LNO-LAO and LTNAO superlattices. The resulting polarization-dependent spectra are shown in Fig. 3. In the two-component superlattice [Fig. 3(a)], no significant dichroic signal is observed. We ascertain $r=1.02 \pm 0.05$ from Eq. (1), consistent with our DFT calculations and previous reports on $\mathrm{LaNiO}_{3}-\mathrm{LaAlO}_{3}$ superlattices [23,24]. In contrast, there is a marked dichroism for the LTNAO superlattice [Fig. 3(b)]. The hole ratio computed from the data is $r=0.55 \pm 0.04$. This result represents a $\sim 50 \%$ reduction in $r$ compared to the bulk and the largest experimentally observed orbital polarization in perovskite nickelate systems to date. Furthermore, the data agree well with the DFT predictions, indicating that the synthesized LTNAO superlattice possesses the theoretically calculated nondegenerate band structure and that it can be tuned to have a single band near the Fermi level.

For a quantitative comparison with previous strategies of orbital degeneracy breaking, namely epitaxial strain and confinement, one cannot directly compare the hole ratios
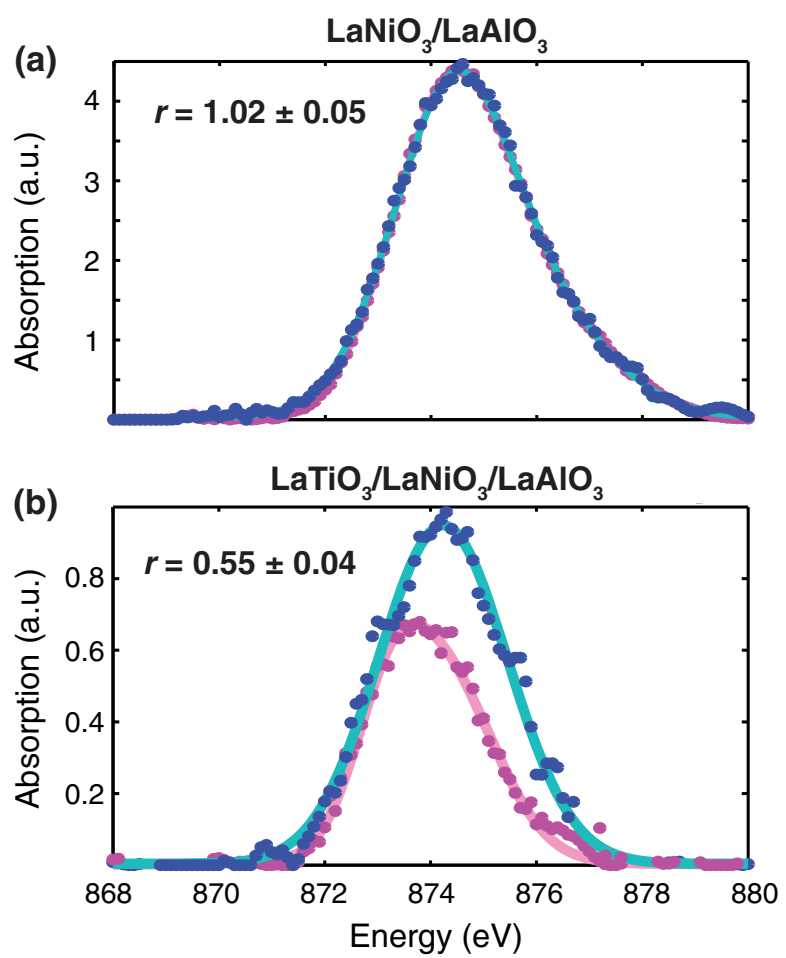

FIG. 3 (color online). Measured x-ray absorption (circles) for in-plane (blue) and out-of-plane (pink) polarizations of (a) twocomponent and (b) three-component nickelate superlattices. The solid colored lines are double Gaussian fits used in evaluating Eq. (1).

observed here with previous experiments that were designed to shift the $x^{2}-y^{2}$ orbitals to lower energy (i.e., flipping the $e_{g}$ configuration). In such systems, the ideal value for the hole ratio (corresponding to a half filled $x^{2}-y^{2}$ orbital and a completely unoccupied $3 z^{2}-r^{2}$ orbital) would be $r=2$. The best reported orbital polarization in such perovskite nickelate-based structures has a value of $r \approx 1.19$, which is realized in two-component $\mathrm{LaNiO}_{3}$ superlattices and attributed primarily to the effects of epitaxial strain [28]. This $\sim 20 \%$ change in the orbital occupation may be compared with the $\sim 50 \%$ change we observe in the LTNAO superlattice to reveal a factor of 2-3 improvement on the state of the art [53]. Similarly, in terms of energetic splitting, we find an $e_{g}$ orbital energy splitting of $\Delta_{e_{g}} \sim 0.8 \mathrm{eV}$ for the LTNAO tricomponent structure compared with $\Delta_{e_{g}} \sim 0.3 \mathrm{eV}$ for the strained twocomponent structures.

To provide further context for understanding the orbital degeneracy breaking relative to conventional experimental approaches, we theoretically consider a confined system of $\left(\mathrm{LaNiO}_{3}\right)_{1}-\left(\mathrm{LaAlO}_{3}\right)_{3}$ superlattices with varying degrees of in-plane epitaxial strain. We find that in order to achieve our observed value of $r=0.5$, a compressive strain of $>10 \%$ would be required [37]. This agrees with our assessment of the measured asymmetry of the Ni-O bond environment. Similarly, to raise the $e_{g}$ crystal field splitting 
to $\Delta_{e_{q}} \sim 0.8 \mathrm{eV}$ (our value for LTNAO) requires $>10 \%$ epitaxial strain (extrapolating from Ref. [35]). Instead of relying on limited epitaxial constraints, we impose bond length and crystal field distortions by using polar fields, resulting from internal charge transfer, to drive ionic distortions. We note that this mechanism has conceptual similarities to a recently proposed "electrostatic strain" approach that uses cation ordering in Ruddlesden-Popper structures to influence the Ni-O bond distortions [34]; however, the magnitude of the effect in our LTNAO system, as measured by $d_{\mathrm{ap}} / d_{\mathrm{inp}}$ and $\Delta_{e_{g}}$, is both widely tunable and appreciably larger, as explained below.

One aspect of the LTNAO system that can be tuned is the insulator in the heterostructure. To illustrate this tunability, we theoretically consider a series of nickelate superlattices of the type $\left[\left(\mathrm{LaTiO}_{3}\right)_{1}\right.$ - $\left(\mathrm{LaNiO}_{3}\right)_{1}$-(insulator)] with different insulators [37]. The calculations demonstrate that modifying the insulating barrier has a dramatic effect on the Ni bonding environment. For instance, the average apical to in-plane $\mathrm{Ni}$-anion bond length ratio ranges from $d_{\text {ap }} / d_{\text {inp }}=1.14$ to $d_{\text {ap }} / d_{\text {inp }}=1.45$ when changing the insulator from $\mathrm{LaAlO}_{3}$ to $\mathrm{BaO}$. Such large bond length modifications considerably alter the crystal field of the $\mathrm{Ni}$ and thus affect the orbital configuration in kind. Hence, we find a correlation between the average $\mathrm{Ni}$-anion bond length ratio and the hole ratio, with larger $d_{\mathrm{ap}} / d_{\text {inp }}$ giving smaller $r$. The hole ratio can be reduced to $r=0.18$ by using $\mathrm{RbF}$ as an insulator and upon electron doping (25\% $\mathrm{Ba}$ for $\mathrm{Rb}$ ), decreases even further to $r=0.09$, approaching the value for the undoped cuprates $[54,55]$. In addition, one can further fine-tune the orbital polarization using strain by choice of substrate. These results demonstrate that our methodology allows one to access a wide and relatively continuous range of $r$, and provides unique control over orbital configuration.

The LTNAO superlattice described here is one of a promising new class of materials that show robust and large orbital polarizations due to symmetry breaking, internal charge transfer, and apical oxygen bond elongation from built-in polar fields. The experimental realization of the LTNAO structure, and confirmation of its predicted atomic and electronic structure, provides a template to achieve widely tunable orbital configurations. With the various control parameters, one is able to tune the symmetry of the electronically active bands ranging from and between those of $\mathrm{Ni}$ - and $\mathrm{Cu}$-based oxides. This approach represents a robust and realizable path to emulating cuprate-like physics in nickelates, as has been proposed previously [14,21-30,35]. More generally, this method, which relies on simple physical principles, represents a new avenue for experiment and theory to explore low-energy phenomena and orbital-dependent properties of diverse materials systems.

This work was supported by NSF MRSEC DMR 1119826 (CRISP). Use of the Advanced Photon Source is supported by the U.S. Department of Energy, Office of Basic Energy Sciences under Contract No. DE-AC0206CH11357. Use of the National Synchrotron Light Source, Brookhaven National Laboratory, is supported by the U.S. Department of Energy, Office of Science, Office of Basic Energy Sciences, under Contract No. DEAC02-98CH10886. Computational facilities are supported by NSF Grant No. CNS 08-21132 and by the facilities and staff of the Yale University Faculty of Arts and Sciences High Performance Computing Center. Additional computations are carried out via the NSF XSEDE resources through Grant No. TG-MCA08X007. H. C. is supported by NSF Grant No. DMR 1120296.

* Corresponding author. ankit.disa@yale.edu

[1] T. V. Menshchikova, M. M. Otrokov, S. S. Tsirkin, D. A. Samorokov, V. V. Bebneva, A. Ernst, V. M. Kuznetsov, and E. V. Chulkov, Nano Lett. 13, 6064 (2013).

[2] J. Suntivich, H. A. Gasteiger, N. Yabuuchi, H. Nakanishi, J. B. Goodenough, and Y. Shao-Horn, Nat. Chem. 3, 546 (2011).

[3] J. S. Lee, Y. W. Xie, H. K. Sato, C. Bell, Y. Hikita, H. Y. Hwang, and C. C. Kao, Nat. Mater. 12, 703 (2013).

[4] K. Yoshimatsu, K. Horiba, H. Kumigashira, T. Yoshida, A. Fujimori, and M. Oshima, Science 333, 319 (2011).

[5] E. J. Monkman, C. Adamo, J. A. Mundy, D. E. Shai, J. W. Harter, D. Shen, B. Burganov, D. A. Muller, D. G. Schlom, and K. M. Shen, Nat. Mater. 11, 855 (2012).

[6] E. Pavarini, I. Dasgupta, T. Saha-Dasgupta, O. Jepsen, and O. Andersen, Phys. Rev. Lett. 87, 047003 (2001).

[7] N. B. Aetukuri et al., Nat. Phys. 9, 661 (2013).

[8] J. B. Goodenough, Chem. Mater. 26, 820 (2014).

[9] H. Y. Hwang, Y. Iwasa, M. Kawasaki, B. Keimer, N. Nagaosa, and Y. Tokura, Nat. Mater. 11, 103 (2012).

[10] Y. Tokura and Y. Tomioka, J. Magn. Magn. Mater. 200, 1 (1999).

[11] G. Maris, Y. Ren, V. Volotchaev, C. Zobel, T. Lorenz, and T. Palstra, Phys. Rev. B 67, 224423 (2003).

[12] L. F. Feiner, M. Grilli, and C. Di Castro, Phys. Rev. B 45, 10647 (1992).

[13] J. Chakhalian, J. W. Freeland, H. U. Habermeier, G. Cristiani, G. Khaliullin, M. van Veenendaal, and B. Keimer, Science 318, 1114 (2007).

[14] A. V. Boris et al., Science 332, 937 (2011).

[15] R. Eguchi, A. Chainani, M. Taguchi, M. Matsunami, Y. Ishida, K. Horiba, Y. Senba, H. Ohashi, and S. Shin, Phys. Rev. B 79, 115122 (2009).

[16] D. P. Kumah, A. S. Disa, J.H. Ngai, H. Chen, A. Malashevich, J. W. Reiner, S. Ismail-Beigi, F.-J. Walker, and C. H. Ahn, Adv. Mater. 26, 1935 (2014).

[17] M. L. Medarde, J. Phys. Condens. Matter 9, 1679 (1997).

[18] R. Scherwitzl, S. Gariglio, M. Gabay, P. Zubko, M. Gibert, and J. M. Triscone, Phys. Rev. Lett. 106, 246403 (2011).

[19] J. Son, P. Moetakef, J. M. LeBeau, D. Ouellette, L. Balents, S. J. Allen, and S. Stemmer, Appl. Phys. Lett. 96, 062114 (2010). 
[20] J. B. Torrance, P. Lacorre, A. I. Nazzal, E. J. Ansaldo, and C. Niedermayer, Phys. Rev. B 45, 8209 (1992).

[21] J. Chaloupka and G. Khaliullin, Phys. Rev. Lett. 100, 016404 (2008).

[22] P. Hansmann, X. Yang, A. Toschi, G. Khaliullin, O. K. Andersen, and K. Held, Phys. Rev. Lett. 103, 016401 (2009).

[23] E. Benckiser et al., Nat. Mater. 10, 189 (2011).

[24] J. W. Freeland, L. Jian, M. Kareev, B. Gray, J. W. Kim, P. Ryan, R. Pentcheva, and J. Chakhalian, Europhys. Lett. 96, 57004 (2011).

[25] M. J. Han, C. A. Marianetti, and A. J. Millis, Phys. Rev. B 82, 134408 (2010).

[26] M. J. Han, X. Wang, C. A. Marianetti, and A. J. Millis, Phys. Rev. Lett. 107, 206804 (2011).

[27] J. Liu, S. Okamoto, M. van Veenendaal, M. Kareev, B. Gray, P. Ryan, J. W. Freeland, and J. Chakhalian, Phys. Rev. B 83, 161102 (2011).

[28] M. Wu et al., Phys. Rev. B 88, 125124 (2013).

[29] H. Chen, D. P. Kumah, A. S. Disa, F. J. Walker, C. H. Ahn, and S. Ismail-Beigi, Phys. Rev. Lett. 110, 186402 (2013).

[30] H. Chen, A. J. Millis, and C. A. Marianetti, Phys. Rev. Lett. 111, 116403 (2013).

[31] H. N. Lee, H. M. Christen, M. F. Chisholm, C. M. Rouleau, and D. H. Lowndes, Nature (London) 433, 395 (2005).

[32] S. M. Nakhmanson, K. M. Rabe, and D. Vanderbilt, Appl. Phys. Lett. 87, 102906 (2005).

[33] H. Yamada, M. Kawasaki, Y. Ogawa, and Y. Tokura, Appl. Phys. Lett. 81, 4793 (2002).

[34] P. V. Balachandran, A. Cammarata, B. B. Nelson-Cheeseman, A. Bhattacharya, and J. M. Rondinelli, APL Mater. 2, 076110 (2014).

[35] O. E. Peil, M. Ferrero, and A. Georges, Phys. Rev. B 90, 045128 (2014).

[36] For illustrative purposes, we show band structures for $1 \times 1$ supercell calculations in Fig. 1. The numerical results discussed in the Letter are based on $c(2 \times 2)$ supercell structures (including octahedral rotations), which agree with experimental measurements.

[37] See Supplemental Material at http://link.aps.org/ supplemental/10.1103/PhysRevLett.114.026801, which includes Refs. [38-50], for additional data and details on sample growth and characterization, DFT calculations, and XLD analysis.
[38] A. S. Disa, D. P. Kumah, J. H. Ngai, E. D. Specht, D. A. Arena, F. J. Walker, and C. H. Ahn, APL Mater. 1, 032110 (2013).

[39] P. Giannozzi et al., J. Phys. Condens. Matter 21, 395502 (2009).

[40] D. Vanderbilt, Phys. Rev. B 41, 7892 (1990).

[41] M. Cococcioni and S. de Gironcoli, Phys. Rev. B 71, 035105 (2005).

[42] G. Gou, I. Grinberg, A. M. Rappe, and J. M. Rondinelli, Phys. Rev. B 84, 144101 (2011).

[43] N. Marzari and D. Vanderbilt, Phys. Rev. B 56, 12847 (1997).

[44] I. Souza, N. Marzari, and D. Vanderbilt, Phys. Rev. B 65, 035109 (2001).

[45] A. A. Mostofi, J. R. Yates, Y.-S. Lee, I. Souza, D. Vanderbilt, and N. Marzari, Comput. Phys. Commun. 178, 685 (2008).

[46] J. Stöhr, NEXAFS Spectroscopy, Springer Series in Surface Sciences (Springer, Berlin; New York, 1996), 1st ed., 25, p. 403.

[47] W. B. Wu et al., J. Electron Spectrosc. Relat. Phenom. 137-140, 641 (2004).

[48] M. Crespin, P. Levitz, and L. Gatineau, J. Chem. Soc., Faraday Trans. 2 79, 1181 (1983).

[49] M. Björck and G. Andersson, J. Appl. Crystallogr. 40, 1174 (2007).

[50] The driving force for this charge transfer can be understood from a chemical perspective by comparing the electronegativities of $\mathrm{Ni}$ and $\mathrm{Ti}$ (1.91 and 1.54, respectively).

[51] Y. Yacoby, M. Sowwan, E. Stern, J. O. Cross, D. Brewe, R. Pindak, J. Pitney, E. M. Dufresne, and R. Clarke, Nat. Mater. 1, 99 (2002).

[52] G. v. d. Laan, J. Phys. Soc. Jpn. 63, 2393 (1994).

[53] Note that, using Eq. (2), with realistic $n_{e_{g}}$ values ranging from 1 to 2 for $\mathrm{LaNiO}_{3}$-based systems, yields $P=8-25 \%$ for Ref. [28] and 29-87\% for the LTNAO superlattice measured here, which shows greater than a factor of 3 improvement in the orbital polarization.

[54] C. T. Chen, L. H. Tjeng, J. Kwo, H. L. Kao, P. Rudolf, F. Sette, and R. M. Fleming, Phys. Rev. Lett. 68, 2543 (1992).

[55] E. Pellegrin et al., Phys. Rev. B 47, 3354 (1993). 